\title{
A Single Institution Study Experience of Secondary Breast Angio- sarcoma after Breast Conserving Treatment: Multidisciplinary Management
}

\author{
Angela Strazzanti, $M D^{1 *}$, Claudio Trovato, $M D^{1}$, Santi Gangi, $M D^{1}$, Dario Marletta, \\ $M D^{2}$, Roberto Milazzotto, $M D^{2}$ and Corrado Spatola, $M D^{2}$
}

${ }^{1}$ Department of General Surgery, Senology University Hospital Policlinico-Vittorio Emanuele, Catania, Italy

${ }^{2}$ Department of Radiotherapy Unit, University Hospital Policlinico-Vittorio Emanuele, Catania, Italy

*Corresponding author: Angela Strazzanti, MD, Departement of General Surgery, Senology University Hospital PoliclinicoVittorio Emanuele, Catania, Italy

\begin{abstract}
The aim of this study, focusing on a small number of cases, is to deal with the angiosarcoma of the breast, representing less than $1 \%$ of all soft tissue breast tumors, and finally share our experience. As a consequence of breast-conserving therapy $(\mathrm{BCT})$ that is the standard treatment nowadays, a new type of angiosarcoma has been highlighted: RadiationInduced Angiosarcoma (RIAS).

Here is reported a retrospective analysis of RIAS patients' characteristic features, together with an account of the integrated treatment and the outcome of six cases of RIAS treated in our institution from 2013 to 2018.

Breast angiosarcoma as a consequence of surgery and radioterapy has been rarely recorded but it is a documented complication of radiation treatment for breast cancer, with a high recurrence rate and poor outcomes.

It remains challenging clinically, radiologically and histologically, and thus a high index of suspicion is required in susceptible patients. Simple mastectomy is the primary treatment option, however, an increasing number of studies on the use of radiotherapy and chemotherapy, each with variable degree of success, is being focused. Re-irradiation can be taken into consideration in order to increase local control, while chemotherapy may be considered for cases in a more advanced stage.
\end{abstract}

\section{Keywords}

Breast angiosarcoma (AS), Radiation-induced angiosarcoma (RIAS), Radical mastectomy (RM), Radiation-induced tumors, Adjuvant radiotherapy, Intensity-Modulated Radiotherapy (IMRT)

\begin{abstract}
Abbreviation
RIAS: Radiation-Induced Angiosarcoma; BCT: BreastConserving Therapy; AS: Breast Angiosarcoma; MRI: Magnetic Resonance Imaging; IMRT: Intensity-Modulated Radiotherapy
\end{abstract}

\section{Introduction}

In this work diagnosis, management, and outcome of RIAS in a small number of patients with breast cancer are being discussed.

As a very rare vascular tumor, breast angiosarcoma (AS) can be divided into primary, if it arises without a known precursor, or secondary, if it occurs at the site of previously irradiated skin, in which case it is known as a Radiation-Induced Angiosarcoma (RIAS) of the breast [1]. But, still, no evidence-based guidelines concerning the ideal treatment of angiosarcomas exist.

In those patients undergoing breast conserving surgery with adjuvant radiotherapy, the estimated incidence of RIAS varies between 0.05 and $0.3 \%$, accounting for less than $1 \%$ of breast tumors [1-4] and for approximately about $3 \%$ of all soft-tissue sarcomas.

The incidence of RIAS appears to be increasing, perhaps reflecting the long latency period for the development of these tumours following the widespread adoption of adjuvant radiotherapy for breast cancer.

Citation: Strazzanti A, Trovato C, Gangi S, Marletta D, Milazzotto R, et al. (2019) A Single Institution Study Experience of Secondary Breast Angiosarcoma after Breast Conserving Treatment: Multidisciplinary Management. Int J Cancer Clin Res 6:114. doi.org/10.23937/2378-3419/1410114

Accepted: May 06, 2019; Published: May 08, 2019

Copyright: (C) 2019 Strazzanti A, et al. This is an open-access article distributed under the terms of the Creative Commons Attribution License, which permits unrestricted use, distribution, and reproduction in any medium, provided the original author and source are credited. 
The diagnosis of RIAS of the breast is often delayed because of its benign appearance and the difficulty in differentiating it from the nonspecific skin changes induced by prior radiation or from another disease [5]. The radiological findings are also often nonspecific. Although RIAS typically develops 10 years after radiation treatment for breast cancer, the latency period can range from 6 months to 20 years [6], leading some authors to believe that it is probably being underreported and that the true incidence rates are therefore higher. As the incidence of breast cancer is increasing, the use of breast-conserving surgery followed by radiotherapy has been replacing radical mastectomy as standard treatment. The associated incidence of RIAS is also rising, with an estimated cumulative incidence of 0.9-3.2 per 1000 breast cancer cases [3,4]. In a large population-based cohort study, a history of prior radiotherapy as treatment for breast cancer was associated with 26-fold increase in the risk of developing angiosarcoma when compared with non-irradiated control groups [7]. The prognosis for patients with RIAS remains poor, with 5 -year overall survival rates ranging from 27 to $48 \%$. Despite the fact that RIAS was first described in the early 1920s, its molecular biology is still controversial and there is therefore no targeted therapy available.

Currently, aggressive surgical resection is commonly advocated as the treatment of choice. There is a lack of data proving the efficacy of adjuvant chemotherapy [8].

Surgery, in the form of wide excision or mastecto$\mathrm{my}$, is the mainstay of management in localised disease. Some studies have reported an association between RO margins and improved survival rate, although it has not been demonstrated to be independent of other biological factors, such as tumour size $[9,10]$. There is some evidence that neoadjuvant chemotherapy may improve outcomes in the treatment of the angiosarcoma, the rarity of which limits our knowledge to case reports or small retrospective case series $[5,11,12]$.

In order to characterize a population of patients with RIAS, treated at our hospital for a 5-year period, we have performed a retrospective study analyzing the usefulness of mammography, ultrasound, and magnetic resonance imaging (MRI) for our diagnosis [13].

Our work has been reported in line with the SCARE criteria [14].

\section{Materials and Methods}

Our case series regards patients with histologically proven angiosarcoma of the breast, developed after radiotherapy for breast cancer, between 2013 and 2018, and analyses treatment strategies with a multidisciplinary approach.

The affected patients, six females with radiation-induced breast angiosarcoma, were identified through a comprehensive search in the aforementioned period of the cancer registry of our institution, at University Hospital of Catania. Mean age was 70, range: 59-79 years old. Patients had performed the previous diagnostic and therapeutic management at various institutions, so that the information is not all available. Description of patients' characteristics and treatment procedures per-

Table 1: Patients' characteristics and treatment procedures.

\begin{tabular}{|c|c|c|c|c|c|c|c|c|}
\hline Patient & Age & $\begin{array}{l}\text { Tumor } \\
\text { side }\end{array}$ & $\begin{array}{l}\text { Tumor } \\
\text { histological } \\
\text { characteristics }\end{array}$ & $\begin{array}{l}\text { Years } \\
\text { from initial } \\
\text { treatment }\end{array}$ & Surgery & $\begin{array}{l}\text { Radiotherapy } \\
\text { (timing, dose and } \\
\text { technique) }\end{array}$ & Chemotherapy & Follow-up \\
\hline 1 & 70 & Right & $\begin{array}{l}\text { G3 } \\
\text { Tm }(\max \varnothing 2,7 \\
\mathrm{cm})-\mathrm{R} 0\end{array}$ & 9 & RM & $\begin{array}{l}\text { Immediate RT } \\
50 \mathrm{~Gy} / 20 \mathrm{fr} \\
\text { IMRT }\end{array}$ & $\begin{array}{l}\text { Epi-Ifo } \\
\text { (4 cycles) }\end{array}$ & $\begin{array}{l}\text { NED } \\
\text { Alive }\end{array}$ \\
\hline 2 & 72 & Right & $\begin{array}{l}\mathrm{G} 2 \\
\mathrm{~T} 3,1 \mathrm{~cm}-\mathrm{R} 0\end{array}$ & 6 & RM & $\begin{array}{l}\text { Immediate RT } \\
35 \mathrm{~Gy} / 7 \mathrm{fr} \\
\text { IMRT }\end{array}$ & None & $\begin{array}{l}\text { NED } \\
\text { Alive }\end{array}$ \\
\hline 3 & 79 & Left & $\begin{array}{l}\mathrm{G} 2 \\
\mathrm{Tm}(\max \varnothing 1,5 \\
\mathrm{cm})-\mathrm{R} 0\end{array}$ & 5 & RM & $\begin{array}{l}\text { Immediate RT } \\
40 \mathrm{~Gy} / 20 \mathrm{fr} \\
\text { IMRT }\end{array}$ & None & $\begin{array}{l}\text { Lung/Bone } \\
\text { mets } \\
\text { Alive }\end{array}$ \\
\hline 4 & 59 & Left & $\begin{array}{l}\mathrm{G} 2 \\
\mathrm{~T} 1,6 \mathrm{~cm}-\mathrm{R} 0\end{array}$ & 9 & RM & No RT & $\begin{array}{l}\text { Pac } \\
\text { (12 cycles) }\end{array}$ & $\begin{array}{l}\text { SD } \\
\text { Alive }\end{array}$ \\
\hline 5 & 68 & Right & $\begin{array}{l}\text { G3 } \\
\operatorname{Tm}(\max \varnothing 1,8 \\
\mathrm{cm})-\mathrm{R} 0\end{array}$ & 11 & RM & $\begin{array}{l}\text { Delayed RT } \\
50 \mathrm{~Gy} / 25 \mathrm{fr} \\
\text { IMRT }\end{array}$ & $\begin{array}{l}\text { Epi } \\
\text { (6 cycles) }\end{array}$ & $\begin{array}{l}\text { Lung mets } \\
\text { Died }\end{array}$ \\
\hline 6 & 75 & Left & $\begin{array}{l}\text { G3 } \\
\text { T 2,6 cm - R0 }\end{array}$ & 6 & RM & $\begin{array}{l}\text { Immediate RT } \\
40 \mathrm{~Gy} / 20 \mathrm{fr} \\
\text { IMRT }\end{array}$ & None & $\begin{array}{l}\text { Lung mets } \\
\text { Died }\end{array}$ \\
\hline
\end{tabular}

RM: Radical Mastectomy, Epi: Epirubicin (dose $100 \mathrm{mg} / \mathrm{mq}$ day 1), Ifo: Ifosfamide (dose $2 \mathrm{~g} / \mathrm{mq}$ day 1-3), Pac: Paclitaxel (dose 100 mg/mq weekly), RT: Radiotherapy, IMRT: Intensity-modulated Radiotherapy, fr: Fractions, G: Grading, T: Tumor dimension, Tm: Multiple tumor nodules, R: Status of margins, NED: Non-Evidence of Disease, mets: Distant metastases; SD: Stable Disease. 
formed after RIAS diagnosis are described in Table 1. All patients had undergone breast-conserving treatment at the time of diagnosis, with adjuvant radiotherapy administered by conventional 3-dimensional conformal radiotherapy to a total dose of $50 \mathrm{~Gy}$, with daily fractionation of $2 \mathrm{~Gy}$, with or without a tumor bed boost of $10 \mathrm{~Gy}$. Tumors were right-sided in 3 patients, and left-sided for the other 3. During adjuvant treatment, five out of six patients received anthracycline-based systemic chemotherapy, according to institutional guidelines, while all patients received adjuvant tamoxifen/ aromatase inhibitor hormonal therapy. No patient had HER2-positive cancer at the time of diagnosis.

During follow-up, after a median period of 7,6 years (range: 5-11 years), the diagnosis of ipsilateral breast angiosarcoma was performed: the most frequently exhibited signs by the patients at onset were red-violet coloured skin nodules, located in the context of irradiation fields, with a moderately variable growth rate from a few weeks to 4-6 months. All patients received a histological confirmation of secondary angiosarcoma and a radical mastectomy was performed in all cases. To diagnose RIAS, the Cahan and Arlen criteria were followed [5]: sarcoma arising within the previous irradiated field; a latency period of at least 3 years between radiotherapy and the development of the sarcoma; and a histological distinction between the secondary sarcoma and the primary neoplasm. All six patients received a complete radiological work-up with mammography, ultrasound and MR (in 4/6 patients) in the attempt to confirm the clinical suspicion of RIAS, but no pathognomonic characteristics were identified. The growing interest of scientific literature in identifying specific features of "radiation signature" has not yet produced consolidated data. Three of the six patients highlighted in our case series were the object of an immunohistochemical study for the evaluation of MYC expression, which had shown a high expression on such patients. Table 1 reports also tumor histological characteristics. The date of RIAS diagnosis was defined as the day on which the histological diagnosis was made. The latency period was defined as the time from the first radiation session and the date of RIAS diagnosis. Only one patient showed synchronous metastases (in the lung) at diagnosis of RIAS, while other five patients showed only breast disease.

All patients undergoing surgery at our Hospital were discussed at a sarcoma multidisciplinary meeting in a pre-operative phase. Patients were classified as having resectable disease if pre-operative assessment indicated that a $2 \mathrm{~cm}$ or greater negative margin could be achieved by surgery with or without plastic surgical reconstruction in the form of a single pedicled or free myocutaneous flap. If the desired negative margins required more extensive reconstruction, such as with extensive resurfacing by large skin grafting, the patient would be classified as having irresectable disease.
Rapidly progressive disease, where disease volume increased over a time span of 2-3 weeks from being suitable for mastectomy alone or in combination with a pedicled flap to requiring more extensive reconstruction, was also judged irresectable in oncological terms.

Preoperative biopsies were performed to confirm the presence of AS and exclude other diseases.

Macroscopically complete resection was considered by the operating surgeon. Histologically, the resection was classified as RO (microscopically negative) if the negative margins were more than $1 \mathrm{~cm}$ circumferentially or R1 (microscopically positive) if tumour extended to or within less than $1 \mathrm{~cm}$ of the resection margin.

Four patients were recruited for immediate re-irradiation, while one patient was treated after 13 months when a parietal relapse occurred (she had refused immediate re-irradiation), the last patient was excluded from re-irradiation, as he had achieved dose limits for the heart in the previous treatment. The decision on administration of adjuvant chemotherapy was made on a case-by-case basis, based on the data available in relation to the previous treatments received and the performance status of the patients. One patient received four cycles of epirubicin-ifosfamide chemotherapy, one epirubicin alone for six cycles, three were excluded for medical reasons (age, comorbidities or tolerance dose of the anthracyclines already previously reached). One patient received weekly paclitaxel for 12 cycles for synchronous lung metastases.

Radiotherapy was administered taking into account the doses received from healthy tissues in the previous irradiation and prior administration of anthracyclines. For these reasons, we chose to carry out the re-irradiation by means of intensity-modulated radiotherapy (IMRT, see Figure 1), using a step-and-shoot technique, according to our institutional procedures [2-4]. The Radiation dose and fractionation scheme which were used varied: two patients received conventional fractionation to $50 \mathrm{~Gy}$, two patients received $40 \mathrm{~Gy}$ (because the time elapsed from the previous irradiation was short and the neoplasm was located on the left breast), one patient $35 \mathrm{~Gy}$ in $5 \mathrm{~Gy}$ fraction once a week (due to reduced clinical compliance).

\section{Results}

No positive margins were reported in the patients who had received radical mastectomy performed by the same surgical team.

Following radical mastectomy, patients received treatment as planned, without any interruptions, both for chemotherapy and radiation therapy. At a median follow-up time of 22 years (range: 1.5-4 years), three patients are alive, without evidence of relapse or progressive disease. One patient with synchronous pulmonary metastases at diagnosis is still receiving active on- 


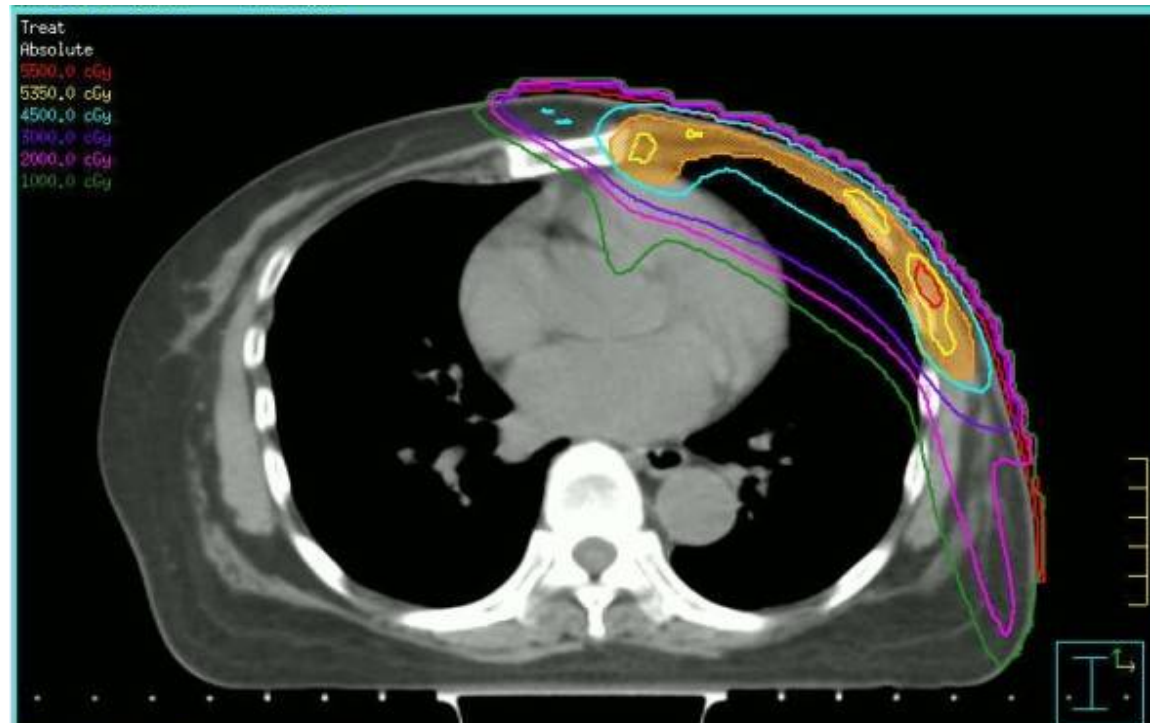

Figure 1: Transverse CT image with step-and-shot intensity-modulated dose distribution.

cological treatment. One patient developed pulmonary and bone metastases, but she is still alive at the time of the work submission. The last two patients developed pulmonary metastases, one of which associated with local recurrence, and died after 8-10 months (distribution of patients characteristics in relation to disease presentation and treatment received is described in Table 1). Overall, disease-free survival was 14 months for five patients without systemic disease at diagnosis of RIAS, while progression-free survival for synchronous metastatic patient at diagnosis was 12 months.

As we may expect, poorer survival outcomes, reported in literature, were noticed in patients presenting with locally advanced disease unsuitable for surgical management compared to the cases in our series. These factors highlight the importance of early diagnosis in RIAS patient. Angiosarcomas often present themselves insidiously with purple or red skin changes and may be easily mistaken for bruising or benign skin changes leading to delayed investigation and diagnosis. Early detection and prompt referral may potentially reduce the number of patients showing irresectable disease and improve both local and distant disease control.

\section{Discussion}

Radiation-induced tumors typically arise in the context or at the edge of the irradiation field where the dose is not uniform and may result lower than that necessary to induce cell destruction, or cause mutations that may lead to the carcinogenesis process. As an effect of ionizing radiation, chemical and biochemical modifications induced in the living organisms produce functional and morphological alterations of the cells causing a biological damage. Every cell, able to replicate, may become potentially cancerous; if the cells that present chromosomal modifications escape normal cellular controls, carcinogenesis may occur.
The probability of developing tumors in patients undergoing radiation therapy for treating breast cancer is very low and cannot limit the use of radiotherapy when indicated. Radiation doses above $50 \mathrm{~Gy}$ induce cell death and lower doses (<30 Gy) cause genetic instability by damaging cell repair mechanisms.

Numerous studies have suggested the correlation between the onset of soft tissue sarcomas and the radiation treatments performed to deal with breast tumors. Radiotherapy increases the risk of developing soft tissue sarcomas and, in particular, of developing angiosarcoma [15]. RIAS represents, approximately, less than $1 \%$ of breast tumors [2]. Radioinduced angiosarcoma has many clinical manifestations: patients often show asymptomatic skin changes that can hardly be distinguished from the common actinic alterations that can be found after the radiotherapy treatment on the breast. Patients often show a palpable swelling, a purple-red area similar to a hematoma. Angiosarcomas can be confused with benign manifestations such as angiomas or atypical telangiectasia. Purple papules or erythematous nodules may also occur.

Despite all therapeutic efforts, the five-year survival rate varies from $27 \%$ to $62.8 \%$ (2-3). Although RIAS typically develops 10 years after radiation treatments for breast cancer, the latency period can range from 6 months to 20 years. Radioinduced angiosarcoma is indistinguishable from spontaneous angiosarcoma but some differences can be assessed: Lae, et al. compared the c-myc amplification on chromosome $8 \mathrm{q} 24.21$ in 32 RASB specimens and 15 sporadic angiosarcoma specimens [16].

In our series, patients experienced non-specific signs of disease, but diagnosis was made promptly in the majority of cases. Latency period was in accordance to that reported in the literature, with a median time of 76 years. 
RIAS is known to have a high recurrence rate. Local recurrence, either in the tumor bed or along the surgical scar, is reportedly detected in a majority (96\%) of cases of RIAS. That is probably due to multifocal growth of the RIAS or remnants of malignant tissue after surgery, even with negative surgical margins. Metastatic disease, most frequently to the lungs and the liver, can be present at the same time or shortly after local recurrences $[7,8]$. Abbott, et al. retrospectively reviewed all cases of RIAS published as of 2017 and summarized the data [17]. The authors found that the local recurrence rate was $59 \%$ and that the median time to recurrence was 6 months (range, 1-78 months). They also found that metastatic disease was usually preceded by at least one local recurrence and that the most common locations were the lungs, the contralateral breast, and the skeleton. Furthermore, their histological review of 42 cases suggested that poorly differentiated RIAS was associated with a high risk of metastasis, whereas welldifferentiated tumors presented a higher risk for local recurrence rather than for distant metastases.

Radiation-induced sarcoma is a rare but highly aggressive neoplasm. Its management is a matter of debate, because no treatment guidelines are available, the prognosis is poor and 5-year survival rate varies widely, as reported before $[2,3,17]$. Nevertheless, aggressive surgical resection is commonly advocated as the treatment of choice.

Surgery, in the form of mastectomy with or without plastic reconstruction, is the treatment of choice in patients presenting localised disease. In the series of patients under treatment, it is possible to achieve microscopically complete (RO) resection margins in more than $80 \%$. RIAS typically present themselves as multifocal lesions. The tendency of this pathology to create microsatellite deposits may contribute to the difficulty in obtaining local control.

The importance of performing a complete pathological resection has been stressed in the literature, although no standard guidelines regarding the recommended distance of clearance have been published. In the current series, those who developed local recurrence were found to have closer margins than those who did not. However, marginal status was not found to be independently prognostic of oncological outcomes in this series. This would suggest that the ability to achieve greater margins depends on other biological tumor factors that also determine the outcome, such as the size. It is likely that tumor biology is mainly responsible for the outcome in RIAS, although the initial surgery should aim for macroscopic clearance, it should be cautioned that achieving greater negative margins does not necessarily equate to improved patient outcomes.

Despite the increasing number of studies focusing on chemotherapy and radiotherapy, whether neoadjuvant or adjuvant, their role remains unclear. There is a lack of data proving the efficacy of adjuvant chemotherapy, recent data have suggested that radiotherapy can be useful in preventing recurrences [18]. Adjuvant radiotherapy after surgery, according to Johnstone, et al. allows for better local control [19]. Role of IMRT in the treatment of chest wall is currently under evaluation, because it can reduce the incidence rate of radiation toxicity by decreasing organs at risk (OARs) irradiation. In a dosimetry comparison study, authors report that IMRT plan to the chest wall and target, compared with conventional treatments, permits to lower the maximum dose, increase the minimum dose, thus resulting in better conformity and uniformity of the target volume [20].

The role and efficacy of chemotherapy has not been established as the available body of data comes from small size sample groups, from which definite conclusions cannot be drawn, but probably patients with higher malignancy grade, G3 and with recurrence, could benefit most from neo or adjuvant chemotherapy [21].

The use of neo/adjuvant chemotherapy was also found to be associated with improved local disease control in a large retrospective series of patients with radiation-induced sarcomas of all sites, although not associated with improved rates of systemic relapse or survival [22]. Adjuvant chemotherapy was not found to produce a benefit in terms of local control or overall survival study of high-risk soft-tissue sarcomas treated with surgery and radiation [23-26]. As such, there is limited evidence to suggest that neo/adjuvant chemotherapy produces a survival benefit in RIAS, although it certainly may be of use as an induction therapy prior to surgery in those presenting with locally advanced disease and may offer patients effective disease palliation in addition. Targeted therapies may offer an alternative treatment in patients with progressive disease.

In our series, as a consequence of the high tendency towards local recurrence of this neoplasm, we have decided to propose all patients a second irradiation, except for one of them for the above-mentioned dosimetric problems. One patient refused immediate re-irradiation and the treatment was carried out at relapse. It was not possible to program a homogeneous radiant treatment for all patients, due to the heterogeneity of the sample for previously used irradiation doses, poor clinical compliance in one case and, above all, lack of clear indications of the literature and the guidelines. In any case, we decided to deliver the treatment by means of an intensity-modulated technique, in order to allow an adequate dose distribution, a reduction of dose exposure of the surrounding healthy tissues, especially for the underlying pulmonary parenchyma and the heart.

\section{Conclusions}

Radiotherapy in the treatment of breast cancer is associated with an increased risk of subsequent 
sarcoma, but the percentage of risk is very low.

RIAS is a rare, aggressive soft-tissue lesion with limited treatment options and high-rates of both local and systemic relapse.

Careful observation after adjuvant radiotherapy is required. Standard treatment involves surgery with simple mastectomy and adjuvant radiotherapy. The chemotherapy may have a role in downsizing locally advanced disease although has no proven effect on survival.

Angiosarcoma is significantly more prevalent in cases treated with radiotherapy, occurring especially in or adjacent to the radiation field. The small difference in risk of subsequent sarcoma for breast cancer patients receiving radiotherapy does not supersede the benefit of radiotherapy.

However, our series is too small to draw final conclusions and this is a limitation of the study. Further evaluation is necessary, also through a larger number of case studies, in order to have a better classification of the pathology and allow a more accurate multidisciplinary management.

\section{Conflicts of Interest}

None of the Authors have any conflicts of interest to disclose.

\section{Sources of Funding}

None.

\section{Ethical Approval}

Approval has been given by the University of Catania ethics committee.

\section{Informed Consent}

Written informed consent was obtained from the patient for publication of this case report and accompanying images. A copy of the written consent is available for review by the Editor-in-Chief of this journal on request.

\section{Author Contributions}

Trovato Agata: Reviewing and editing the article.

\section{Registration of Research Studies}

None.

\section{Disclosure}

None of the authors have anything to disclose.

\section{References}

1. Arora TK, Terracina KP, Soong J, Idowu MO, Takabe K (2014) Primary and secondary angiosarcoma of the breast. Gland Surg 3: 28-34.

2. Torres KE, Ravi V, Kin K, Yi M, Guadagnolo BA, et al (2013) Long-term outcomes in patients with radiationassociated angiosarcomas of the breast following surgery and radiotherapy for breast cancer. Ann Surg Oncol 20: $1267-1274$

3. Kirova YM, Vilcoq JR, Asselain B, Sastre-Garau X, Fourquet A (2005) Radiation-induced sarcomas after radiotherapy for breast carcinoma: a large-scale singleinstitution review. Cancer 104: 856-863.

4. Morgan EA, Kozono DE, Wang Q, Mery CM, Butrynski JE, et al. (2012) Cutaneous radiation-associated angiosarcoma of the breast: poor prognosis in a rare secondary malignancy. Ann Surg Oncol 19: 3801-3808.

5. Zemanova M, Machalekova K, Sandorova M, Boljesikova E, Skultetyova M, et al. (2013) Clinical management of secondary angiosarcoma after breast conservation therapy. Rep Pract Oncol Radiother 19: 37-46.

6. Sheth GR, Cranmer LD, Smith BD, Grasso-Lebeau L, Lang JE (2012) Radiation-induced sarcoma of the breast: a systematic review. Oncologist 17: 405-418.

7. Huang J, Mackillop WJ (2001) Increased risk of soft tissue sarcoma after radiotherapy in women with breast carcinoma. Cancer 92: 172-180.

8. Seinen JM, Styring E, Verstappen V, Vult von Steyern F, Rydholm A, et al. (2012) Radiation-associated angiosarcoma after breast cancer: high recurrence rate and poor survival despite surgical treatment with R0 resection. Ann Surg Oncol 19: 2700-2706.

9. Jallali N, James S, Searle A, Ghattaura A, Hayes A, et al. (2012) Surgical management of radiation-induced angiosarcoma after breast conservation therapy. Am J Surg 203: 156-161.

10. Lindet C, Neuville A, Penel N, Lae M, Michels JJ, et al. (2013) Localised angiosarcomas: the identification of prognostic factors and analysis of treatment impact. A retrospective analysis from the French Sarcoma Group (GSF/GETO) Eur J Cancer 49: 369-376.

11. Alvarado-Miranda A, Bacon-Fonseca L, Ulises LaraMedina F, Maldonado-Martinez H, Arce-Salinas C (2013) Thalidomide combined with neoadjuvant chemotherapy in angiosarcoma of the breast with complete pathologic response: case report and review of literature. Breast Care 8: 74-76.

12. Oxenberg J, Khushalani NI, Salerno KE, Attwood K, Kane JM 3rd (2015) Neoadjuvant chemotherapy for primary cutaneous/ soft tissue angiosarcoma: determining tumor behavior prior to surgical resection. J Surg Oncol 111: 829-833.

13. Strazzanti A, Trovato C, S Gangi, N Pacini, F Basile (2018) Contralateral lymph node metastasis in a woman with new primary breast cancer: Systemic desease or locoregional diffusion? Int J Surg Case Rep 53: 400-402.

14. Agha RA, Fowler AJ, Saeta A, Barai I, Rajmohan S, et al. (2016) The SCARE Statement: Consensus-based surgical case report guidelines. Int J Surg 34: 180-186.

15. Mery CM, George S, Bertagnolli MM, Raut CP (2009) Secondary sarcomas after radiotherapy for breast cancer: sustained risk and poor survival. Cancer 115: 4055-4063.

16. Laé M, Lebel A, Hamel-Viard F, Asselain B, Trassard M, et al. (2015) Can c-myc amplification reliably discriminate postradiation from primary angiosarcoma of the breast? Cancer Radiother 19: 168-174.

17. Abbott R, Palmieri C (2008) Angiosarcoma of the breast following surgery and radiotherapy for breast cancer. Nat Clin Pract Oncol 5: 727-736.

18. Monroe AT, Feigenberg SJ, Mendenhall NP (2003) 
Angiosarcoma after breast-conserving therapy. Cancer 97 1832-1840.

19. Johnstone PA, Pierce LJ, Merino MJ, Yang JC, Epstein AH, et al. (1993) Primary soft tissue sarcomas of the breast: local-regional control with post-operative radiotherapy. Int $J$ Radiat Oncol Biol Phys 27: 671-675.

20. Yang B, Wei XD, Zhao YT, Ma CM (2014) Dosimetric evaluation of integrated IMRT treatment of the chest wall and supraclavicular region for breast cancer after modified radical mastectomy. Med Dosim 39: 185-189.

21. Pervaiz N, Colterjohn N, Farrokhyar F, Tozer R, Figueredo A, et al. (2008) A systematic meta-analysis of randomized controlled trials of adjuvant chemotherapy for localized resectable soft-tissue sarcoma. Cancer 113: 573-581.

22. Woll PJ, Reichardt $P$, Le Cesne A, Bonvalot S, Azzarelli A, et al. (2012) Adjuvant chemotherapy with doxorubicin, ifosfamide, and lenograstim for resected soft-tissue sarcoma (EORTC 62931): a multicentre randomised controlled trial. Lancet Oncol 13: 1045-1054.

23. Strazzanti A, Trovato C, Gangi S, Basile F (2018) Breast tuberculosis cases rising in Sicily. Int J Surg Case Rep 53: 9-12.

24. Spatola C, Tocco A, Milazzotto R, Pagana A, Chillura I, et al. (2016) Role, timing and technique of radiotherapy in pediatric pleuropulmonary synovial sarcoma. Future Oncol 12: 73-77.

25. Spatola C, Militello C, Tocco A, Salamone V, Raffaele L, et al. (2016) Intensity-modulated radiotherapy for relapsed malignant pleural mesothelioma. Future Oncol 12: 67-71.

26. Spatola C, Militello C, Tocco A, Salamone V, Luigi R, et al. (2018) Single-institution experience of intensity-modulated radiotherapy for malignant pleural mesothelioma at University of Catania. Future Oncol 14: 17-21. 